\title{
Perceptual and conceptual training mediate the verbal overshadowing effect in an unfamiliar domain
}

\author{
JOSEPH M. MELCHER \\ St. Cloud State University, St. Cloud, Minnesota \\ and \\ JONATHAN W. SCHOOLER \\ University of Pittsburgh, Pittsburgh, Pennsylvania
}

\begin{abstract}
Describing memory for a nonverbal perceptual stimulus can impair recognition. This verbal overshadowing effect may occur when perceptual expertise exceeds verbal expertise, but previous evidence for this hypothesis has been strictly correlational. We conducted two experiments, exploring the relation between expertise and verbal overshadowing by manipulating perceptual and conceptual knowledge. Participants received perceptual training (categorizing mushrooms), conceptual training (lecture), or no training, then viewed a mushroom and described it or not. A recognition test followed. In Experiment 1, perceptual training increased susceptibility to verbalization relative to the no training condition. However, conceptual training did not insulate individuals from the verbal overshadowing effect. Experiment 2 was modified to enhance both forms of training. It replicated the finding that perceptual training increases vulnerability to verbal overshadowing, and indicated that, when sufficiently emphasized, verbalization may be useful following conceptual training. The results demonstrate the role that different forms of learning and expertise may play in mediating language influences on recognition memory.
\end{abstract}

Although verbalization often benefits thought processes, a decade of research has documented some disruptive effects of articulating nonverbal cognition in an array of domains, including perception, memory, and problem solving. Schooler and Engstler-Schooler (1990) first documented these disruptive effects of verbalization, termed verbal overshadowing, for visual memories (faces and colors). In the prototypical experiment, people who see a difficult-to-describe face and describe it from memory are less likely to correctly recognize that face than are people who do not describe it. In addition to being replicated for faces (for a recent meta-analysis, see Meissner \& Brigham, 2001), verbal overshadowing is observed with other forms of visual memory, including visual forms (Brandimonte,

\footnotetext{
This work was based on a dissertation conducted by J.M.M. under the supervision of J.W.S. J.M.M. gratefully acknowledges dissertation support from the University of Pittsburgh Office of Graduate Studies and awards from the American Psychological Foundation and the American Psychological Society Student Caucus Student Research Competition. Hank Mashburn provided expert guidance in developing the mushroom training materials. John Kulas, Michael Anderson, Kathy Pezdek, and an anonymous reviewer offered insightful critiques and suggestions. Portions of this paper have been presented in poster and talk formats at the annual conventions of the American Psychological Society (1999) and the Midwestern Psychological Association (2001). Correspondence concerning this article should be addressed to J.M. Melcher, St. Cloud State University, Department of Psychology, WH 102, St. Cloud, MN 56301-4498 (e-mail: jmelcher@stcloudstate.edu).
}

Hitch, \& Bishop, 1992) and maps (Fiore \& Schooler, 2002) as well as other sensory memory domains including taste (Melcher \& Schooler, 1996), audition (Houser, Fiore, \& Schooler, 2003; Perfect, Hunt, \& Harris, 2002), and nonmnemonic areas such as affective decision making (Wilson \& Schooler, 1991), insight problem solving (Schooler, Ohlsson, \& Brooks, 1993), visual reasoning (DeShon, Chan, \& Weissbein, 1995), and analogical transfer (Sieck, Quinn, \& Schooler, 1999).

One explanation for the verbal overshadowing effect is that verbalization causes individuals to draw on verbal knowledge at the expense of nonverbal knowledge. Thus, when individuals' verbal knowledge about a stimulus is commensurate with their nonverbal knowledge, verbalization may be benign or even beneficial. However, when individuals' perceptually and nonverbally oriented experience is more highly developed than their conceptually and verbally oriented knowledge, this may set up conditions for verbal overshadowing to occur. Studies based on preexisting levels of expertise have found evidence that differing levels of conceptual and perceptual expertise can mediate the verbal overshadowing effect. Fallshore and Schooler (1995) found that verbalization impairs own-race face recognition, hypothesized to rely especially on nonverbal holistic processing (e.g., Rhodes, Brake, \& Atkinson, 1993), without impairing other-race face recognition, hypothesized to rely more on verbal/featural processing (Diamond \& Carey, 1986). More recently, Ryan and Schooler 
(1998) found that face recognition among individuals with strong perceptual skills but weak verbal abilities was particularly disrupted by verbalization. In contrast, individuals with relatively weak perceptual memory skills but strong verbal abilities benefited from verbalization.

One challenge in examining the relation between expertise and verbal overshadowing of faces is that few people (with the possible exception of police artists) have much verbal expertise with faces. Melcher and Schooler (1996) therefore examined the relation between verbal overshadowing and expertise in a perceptual domain in which some individuals have substantial domain-specific verbal expertise: wine tasting. They found that individuals who had perceptual expertise (as assessed by frequency of drinking red wine) but lacked verbal expertise (as indicated by a lack of formal training) showed impaired recognition for a target wine whose taste they had just described. In contrast, wine experts (who had both perceptual experience and formal verbal training) and novices (who had neither form of expertise) were not significantly affected by verbalization. These results are thus consistent with the notion that verbal overshadowing occurs when perceptual expertise is high and verbal expertise is low, but not when either both are high or both are low.

Because previous studies of the relation between verbal overshadowing and expertise have relied on preexisting expertise, we do not know for certain whether expertise per se produced the verbalization effects, or whether they were due to another variable correlated with expertise. To more definitively determine the role of expertise in mediating verbal overshadowing, it is critical to experimentally manipulate expertise through training. If training can mediate verbal overshadowing effects in a manner comparable to that observed with preexisting levels of expertise, then we can have more confidence in its defining role in determining the effects of verbalization. Toward this end, the present studies provided participants with either perceptually or conceptually oriented training in an unfamiliar perceptual domain - mushrooms - in order to determine whether susceptibility to verbal overshadowing depends on the nature of expertise.

\section{Perceptual Versus Conceptual Learning}

Central to the premise that different forms of training might lead to different effects of verbalization is the presumption that individuals can acquire perceptual knowledge in multiple ways. A range of research supports the notion that perceptual knowledge can be acquired through either explicit verbal learning or implicit nonverbal associative learning (cf. Goldstone, 1998; Hall, 1991; Reber, 1993). For example, when verbal description is made difficult or impossible with nonrepresentational stimuli such as random dot patterns or mutilated checkerboards ${ }^{1}$ or by rapid presentation, humans and animals (e.g., pigeons) exhibit strikingly similar perceptual discrimination learning performance (e.g., Mackintosh, 1995; McLaren, Leevers, \& Mackintosh, 1994). However, compared to these learn- ing conditions, people learn substantially faster when they are allowed to engage in hypothesis testing (Mackintosh, 1995). This suggests that humans may rely on either associative or rule-based learning, depending on the precise learning conditions.

In a related vein, Ahissar and Hochstein's (1997; Ahissar, 1999) studies of perceptual learning under difficult conditions suggests that perceptual learning is based on lower level neural processes when the stimuli are presented rapidly and involve relatively fine discriminations, but when stimuli are presented relatively slowly and when the discriminations are initially easier, perceptual learning takes place at higher (i.e., cognitive) levels. In support of this model, they found that exposure to a single "easy" (long exposure) stimulus markedly improved subsequent perceptual discrimination performance (involving very brief exposures), compared to participants who did not get the easy preexposure. In sum, when perceptual information is complex, nonverbalizable, and/or encoded quickly or under load, the resulting mental representation of the stimulus is relatively unlikely to include verbal labels or an organizing schema. Nevertheless, humans and animals are sensitive to statistical regularity and covariation among the stimuli and/or between the stimuli and their contexts (Hall, 1991; Holyoak \& Spellman, 1993; Mackintosh, 1995). Most important, humans are able to use this knowledge without an accompanying ability to consciously or accurately report rules or cognitive processes associated with the performance (e.g., Holyoak \& Spellman, 1993; Reber, 1993). In contrast, when perceptual information is presented more schematically, with verbal labels, and intent to acquire underlying category structures, individuals can acquire perceptual knowledge at a more conceptual level, one that more readily lends itself to verbal analysis.

On the basis of this analysis, it seems quite plausible that perceptual expertise in an unfamiliar domain could be developed either through a bottom-up process resulting from repeated brief exposure to complex visual forms, or through a conceptual framework incorporating verbal labels and schematic visual forms. Both types of learning could, in principle, enhance performance on tasks requiring perceptual knowledge, but they should be differentially available for verbalization. Presuming that verbalization specifically disrupts nonverbalizable perceptual knowledge, perceptual performance based on the application of recently acquired low-level nonverbal knowledge should be vulnerable to verbalization. In contrast, perceptual performance based on recently acquired conceptual knowledge should be less vulnerable and might even be enhanced by explicit verbalization.

\section{Experimental Overview}

In the following two experiments, we examined the effects of perceptual and conceptual training on the acquisition of expertise in the unfamiliar domain of mushroom recognition. We used mushrooms for several reasons: (1) We wanted to use a natural category to increase ecological va- 
lidity, in the hope that realism would make the domain more interesting for participants to study than artificial stimuli; (2) mushroom genuses, like most natural categories, have family resemblance structures (e.g., covariances in shape, color, texture) which at least theoretically provide a basis for encouraging relatively nonanalytic, perceptual learning; (3) most verbal overshadowing studies have used natural stimuli (e.g., faces); and (4) because this was a learning manipulation, the domain had to be one about which most participants had little or no prior perceptual exposure or conceptual knowledge.

In both experiments, participants received one of three types of training: no training (no exposure to mushrooms); perceptual training (in the guise of a cover task in which participants had to quickly categorize a set of mushroom photographs (approximately 300 trials in $40 \mathrm{~min}$ ); or conceptual training (a lecture about mushroom structures and visible features, assessed by a quiz). These experiments enabled us to test three critical predictions about the occurrence of the verbal overshadowing effect as mediated by different types of exposure to mushrooms. The predictions were: (1) that verbal overshadowing would not be observed in the no training condition (because participants presumably have neither perceptual nor conceptual skills); (2) that verbal overshadowing would be induced among participants in the perceptual training condition (because the conceptual knowledge that would be helpful to articulating perceptual memory would be underdeveloped); and finally, (3) that verbalization would benefit those in the conceptual training condition (because participants would have the opportunity to gain verbalizable knowledge about mushrooms).

\section{EXPERIMENT 1}

\section{Method}

\section{Participants}

The participants were 416 students from the University of Pittsburgh subject pool. They participated in partial fulfillment of course requirements or for extra credit.

\section{Design}

This was a 3 (training: no training, perceptual, conceptual) $\times 2$ (verbalization: description, no description) between-groups experiment with recognition test performance as the dependent variable.

\section{Materials}

Perceptual training. The perceptual training consisted of a cover task in which participants learned to categorize mushroom species. The stimuli were photographs of mushrooms scanned from various reference books (see Melcher, 2003, for a URL containing full references and color versions of the images in Appendices A and B). They were converted into image files for computer presentation using the Micro Experimental Lab (MEL) software (Psychology Software Tools, 1993). The photographs were displayed in 256 colors at 72 pixels per in. Display dimensions varied according to the proportions of the original photographs; most were approximately $8 \mathrm{~cm}$ wide $\times 10 \mathrm{~cm}$ tall. Each of the six categories (genuses) of mushrooms was represented by seven photographs, each of a different species within the genus (see Appendix A). For example, one category learning set contained seven species of Agaricus and seven species of
Amanita. Within each genus, we selected species that we judged to have a fair amount of perceptual (i.e., family) resemblance. Each photograph was randomly presented in left and right mirror orientations.

Conceptual training. The conceptual training consisted of a 35min videotaped lecture by the first author about the fundamentals of mushroom morphology, life cycle, visible features, and characteristics that experts use to identify and classify mushrooms. The video training was based on mycology texts and a consultation with a mushroom expert. The video incorporated two live mushroom specimens, approximately 12 whole-mushroom photographs, and numerous partial-mushroom photographs and drawings (e.g., stems). The training emphasized overall shape, component structures, surface textures, and some specialized vocabulary used to describe the shapes and features of the mushrooms. The idea was to provide a schema that the verbalization participants could use during the description and recognition tasks. Each participant received a booklet containing a detailed outline of the lecture. We assessed learning with a 25-point quiz on the lecture material.

Recognition test. The recognition test had three trials, each consisting of a study image and its test arrays (see Appendix B). The target was a different photo of the same species as the studied mushroom. Most of the foils were from the same genus as the target and were selected to have fairly close resemblances to the target. For the perceptual training group, the recognition tests followed the same order as the training trial blocks. That is, if the first block of categorylearning trials included the genus Lepiotaceae, the first recognition test involved a Lepiota target. The order of the three recognition tests was counterbalanced.

\section{Procedure}

No training. These participants simply took the three-trial recognition test (described below, because it was the same for all participants).

Perceptual training. After seating these participants at computer monitors, the experimenter explained the task and talked them through the first few trials of a practice set. The practice set consisted of two small categories (three photos each) of two genuses not subsequently used. On each trial, the MEL program randomly selected a photograph from one of the categories and displayed it for $4 \mathrm{sec}$. The participants' task was to learn to classify the images into two categories, labeled by different numbers. The experimenter told the participants, "although you must at first guess the category assignments, the feedback will enable you to eventually stop guessing." A constantly visible instruction reminded the participants which keys were currently in use for the categories (e.g., "press 1 or 2"). If $4 \mathrm{sec}$ passed without a response, the participants received a "time out" message and a signal to start the next trial; otherwise, the message said "correct" or "incorrect." The practice trials terminated after the participant made six correct responses in a row.

Each of the three training blocks consisted of 98 self-paced trials; each block used two new categories of mushrooms. The participants finished the trials (about 320, depending on individual pace and how long it took them to reach criterion on the practice trials) in 35 to $45 \mathrm{~min}$, after which they began the recognition tests.

Conceptual training. These participants watched the training video, having been told that they would take a quiz on its contents. They received a page on which to take notes or make sketches. After the quiz, they took the recognition test.

Recognition test (all conditions). The experimenter told the participants to pay close attention to the mushroom to be displayed for $10 \mathrm{sec}$. After the target mushroom disappeared, describers read instructions directing them to write a detailed description of the mushroom. Control participants wrote the names of as many U.S. states or cities as they could. During the interpolated task, the experimenter reminded the participants to work continuously. After $3 \mathrm{~min}$, the monitor displayed recognition instructions - namely, that one of the six arrayed photos showed a different photo of the same 


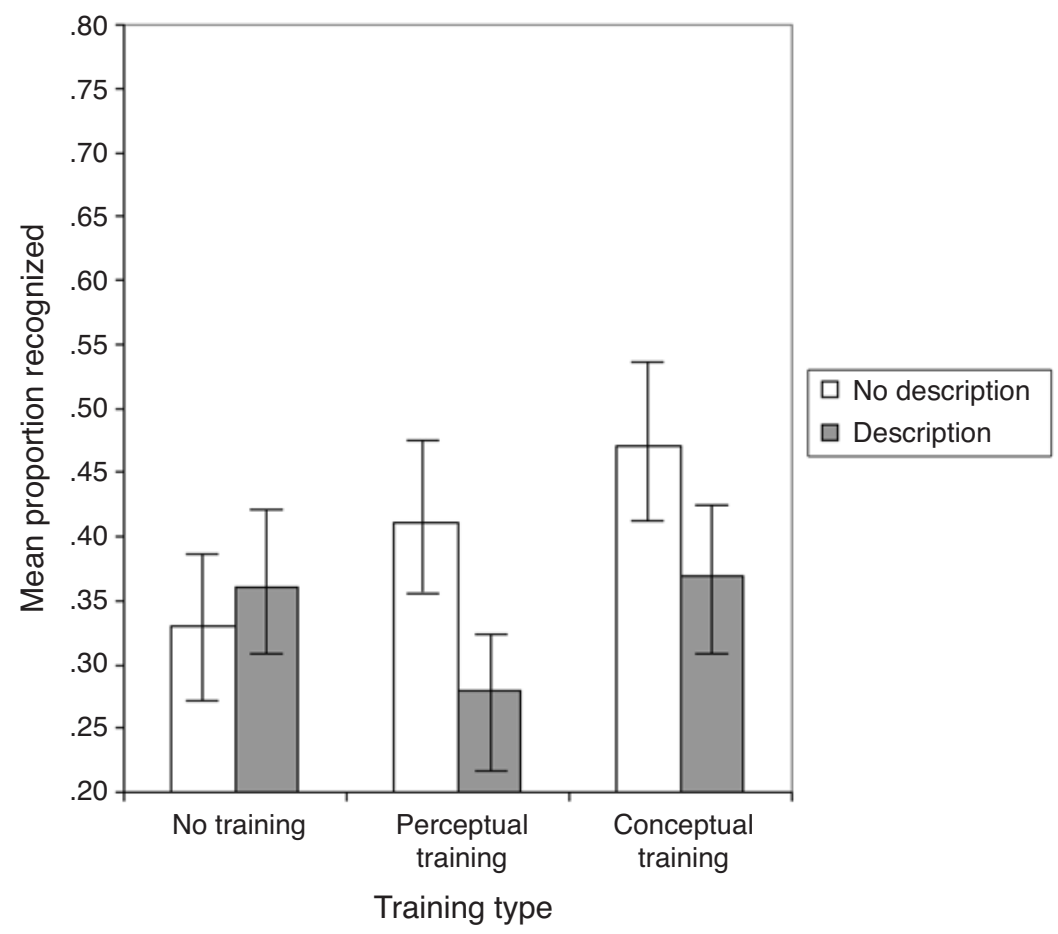

Figure 1. Trial 1 mean recognition accuracy as a function of training and verbalization.

kind of mushroom they had just viewed. The participants indicated their decision by typing its number in the array and then indicated their confidence by typing a number from 1 (guessing) to 7 (very positive).

\section{Results}

Six participants had had fungi training or experience, and three description participants did not write descriptions. Thus, their data were discarded. Figure 1 shows the basic results, including apparent verbal overshadowing effects in both the perceptual and conceptual training groups and a small verbal enhancement effect in the no training condition. (Error bars on all figures indicate $\pm 1 M S_{\mathrm{e}}$.) Virtually all verbal overshadowing research has resulted in a trials effect whereby the verbal overshadowing effect is attenuated or reversed after the first recognition test effect (e.g., Melcher \& Schooler, 1996; Schooler, Ryan, \& Reder, 1996). We also obtained this trials effect in which the numeric verbal overshadowing effects seen in Figure 1 became (nonsignificant) verbal enhancement effects in Trial 2. To test the apparent interaction regarding trial, we did a 2 (verbalization) $\times 2$ (accuracy) $\times 2$ (trial) hierarchical loglinear (HILOG) analysis. HILOG analysis is conceptually similar to factorial analysis of variance (ANOVA), but is more appropriate when the dependent and independent variables are categorical. There was a significant interaction on accuracy involving trial and verbalization $\left[\chi^{2}(1, N=416)=5.26, p=.022\right]$. Therefore, following past practice, we limited our analyses to Trial 1 performance, for which participants were unaffected by knowledge of impending verbalization. ${ }^{2}$

A 3 (training) $\times 2$ (verbalization) $\times 2$ (accuracy) HILOG analysis showed no significant two-way interactions, most notably for training $\times$ verbalization $\left[\chi^{2}(2, N=416)=\right.$ $4.14, p=.13]$. Planned comparisons for differences between the description and control participants within each training condition showed that the apparent verbal overshadowing effect was significant in the perceptual condition $\left[\chi^{2}(1, N=143)=14.16, p=.001(M=.27\right.$ and .42 , respectively)]. The verbal overshadowing effect was nearly significant in the conceptual condition $\left[\chi^{2}(1, N=129)=\right.$ $3.42, p=.065(M=.37$ and .47 , respectively)].

As a manipulation check on the effectiveness of the conceptual training, a random sample of 50 participants ( 25 each from the perceptual training and no training groups) took the mushroom quiz at the end of their sessions. The mean score was 3.26 points $(S D=1.15$, range $1-$ 6 points of 25 possible), compared with the conceptual training mean of $10.5(S D=4.31$; range, $1-22)$. The difference was significant $[t(131)=11.05, p=.000]$. Thus, the training video effectively raised participants' baseline knowledge about mushrooms.

The claim that verbal overshadowing is mediated by expertise implies that the effect of verbalization should depend on how well participants learned from the type of training they received. We therefore separately examined 
the performance of participants who scored high versus low on their respective types of training, based on median splits on the perceptual learning task (mushroom categorization) and conceptual learning task (mushroom knowledge). For the perceptual condition, the median classification accuracy was $72 \%$ on the last 18 (of 98 ) trials of the mushroom classification task $(M=73 \%$; range: $44-98 \%){ }^{3}$ The median score on the mushroom quiz was 9.5 points).

A HILOG analysis of the median splits and Trial 1 recognition accuracy showed that regardless of verbalization, above-median participants in both conditions were significantly more accurate $\left[\chi^{2}(1, N=272)=6.72, p=\right.$ $.01(M=.46$ and .26$)]$. There was no interaction between verbalization and median split, either overall $\left[\chi^{2}(1, N=\right.$ $272)=0.054, p=.81]$ or within conditions: Separate analyses for the perceptual and conceptual learning conditions showed that the median split effect (better learners having better recognition) was driven by the above-median learners in the perceptual training condition $\left[\chi^{2}(1, N=143)=\right.$ $6.06, p=.014]$, but not in the conceptual condition $\left[\chi^{2}(1\right.$, $N=129)=0.07, p=.79]$.

\section{Discussion}

Experiment 1 provided tentative evidence that the nature of recently acquired expertise may mediate the verbal overshadowing effect in an unfamiliar perceptual domain. Specifically, after participants received perceptual training, those who described a previously unseen target mushroom from memory subsequently showed less accurate recognition than did their nondescribing counterparts. The finding that verbalization impaired the recognition performance of those who had received perceptual training in mushroom categorization, while having no effect on untrained participants, conceptually replicates prior findings that expertise mediates verbal overshadowing. This finding is consistent with findings that verbalization impairs own-race recognition but not other-race recognition (Fallshore \& Schooler, 1995) and that verbalization similarly had no significant effect on wine recognition among individuals having little perceptual experience with red wines, while markedly impairing recognition of individuals who regularly drank red wine (Melcher \& Schooler, 1996). Importantly, in contrast to these prior studies, we controlled experience with the stimuli. Thus, we can be more confident that the interactions that we previously observed were a consequence of the perceptual learning and not other variables that might be correlated with experience.

Although the results from the no training and perceptual training conditions are consistent with the differential levels and types of expertise hypothesis, the numericalthough nonsignificant - verbal overshadowing effect in the conceptual training condition was contrary to our prediction of verbal facilitation. Given that conceptual training produced results that resembled (albeit more weakly) those of the perceptual training, one reasonable possibility is that the conceptual training may have disproportionately emphasized perceptual information over con- ceptual information. When familiarizing participants with inherently perceptual stimuli, it is virtually impossible to provide exclusively verbal-conceptual training without at least some perceptual training and exposure. For instance, to have subjects learn what a bulbous stem looks like, it is necessary to show an example. Success in the conceptual training may require participants to adequately consider the conceptual information that is presented with the perceptual elements.

Finally, the conceptual training may have been less effective than the perceptual training. This would explain why the participants failed to benefit (as predicted) by describing the target mushroom's appearance: They may still have lacked (or failed to use) their recently acquired domain-specific verbal skills in a way that could override the verbal overshadowing effect. Experiment 2 addressed these issues by replicating the training procedure of Experiment 1 but with changes designed to enhance training, especially the conceptual training.

\section{EXPERIMENT 2}

Experiment 1 showed that perceptual training mediated the impact of verbalization on the recognition of mushrooms, an unfamiliar natural stimulus. However, we did not observe the predicted verbal enhancement with the conceptual training. In Experiment 2, we attempted to induce verbal enhancement by modifying the conceptual training condition to make participants feel more accountable for learning the conceptual information and to increase the degree to which they processed that information verbally. In Experiment 1, participants in the conceptual training condition were given a blank page and told that it was for taking notes. However, more than a quarter of them took no notes. Prior research has indicated that accountability instructions can substantially increase the motivation and attention that individuals apply to a task (Tetlock \& Kim, 1987). Thus, in Experiment 2, we emphasized the importance of taking notes and asked participants to record their subject number on the note-taking pages. We hypothesized that this modification would encourage participants to take more and better notes and, thus, to form a verbalconceptual knowledge base more useful for the key task of describing the target mushrooms.

In addition, the fact that the verbal overshadowing effect was numerically (though not significantly) stronger among the better perceptual learners suggested that intensifying the perceptual training might bring more participants to some requisite level of perceptual learning. Subject pool limitations also precluded using as many participants and so, in Experiment 2, we intensified the perceptual training by decreasing the number of learning categories while increasing the number of trials in each category. Thus, three blocks of 98 trials were reduced to two blocks of 148 trials each (or $43 \%$ more exposure to the categories). We eliminated the training category and test set for which recognition had been lowest in Experiment 1. 


\section{Method}

Participants. The participants were 235 students from the University of Pittsburgh subject pool.

Design and materials. The design and materials were otherwise identical to those of Experiment 1.

Perceptual training. The number of training (categorization) trials was increased from 98 to 148 trials per species category.

Conceptual training. The participants watched the same training video as in Experiment 1 with the addition of a more explicit injunction to take notes: The experimenter asked the participants to "please write your subject number in the space provided on the sheet provided for taking notes about the video lecture. Your notes will be collected at the end."

Recognition tests. The procedure was identical to that of Experiment 1 , except that there were two instead of three test trials.

Procedure. The procedure was otherwise identical to that in Experiment 1.

\section{Results}

Four participants were dropped because they had had mushroom training $(n=3)$ or did not write a description $(n=1)$. As previously, all analyses were conducted on Trial 1 recognition accuracy.

Figure 2 illustrates the predicted Trial 1 verbal overshadowing effect in the perceptual training condition and a verbal enhancement effect in the conceptual training group (and in the no training group). ${ }^{4}$ A HILOG analysis across all conditions showed a significant training $\times$ verbalization interaction $\left[\chi^{2}(2, N=235)=6.95, p=.031\right]$. As in Experiment 1, we did a median split based on performance in the experimental learning conditions (mushroom image categorization, mushroom quiz score). Figure 3 shows the above-median participants' recognition performance, among whom the verbal overshadowing effect in the perceptual training condition was significant $\left[\chi^{2}(1, N=43)=5.31, p=.01\right.$ (one-tailed) ]. Thus, perceptual learners who excelled on the mushroom categorization test nevertheless fared poorly on the recognition test if they verbalized. Among the above-median learners in the conceptual training condition, the verbal enhancement effect did not quite reach significance $\left[\chi^{2}(1, N=\right.$ 42 ) $=3.20, p=.07$ (two-tailed, because of the nonpredicted result in Experiment 1)].

In contrast, Figure 4 shows the below-median participants' performance. Their overall mean recognition was lower than that of the above-median group (.37 vs. .48), showed a weaker verbal enhancement effect in the conceptual training, and showed barely any verbal overshadowing in the perceptual training condition. The belowmedian group's numeric verbal overshadowing and verbal enhancement effects were not significant $\left[\chi^{2}(1, N=36)\right.$ $\left.=0.02, p=.88 ; \chi^{2}(1, N=37)=2.21, p=.14\right]$.

In order to determine whether there were any differences in description quality between training conditions, we used a communication accuracy test (cf. Fallshore \& Schooler, 1995). All Trial 1 descriptions were transcribed except for misspellings and randomly given to two yoked judges each. The yoked judges were students in an introductory psychology class and a psychology class lab. They viewed the projected recognition array and were asked to choose the mushroom that most resembled the description they received. Each pair of yoked judges was given a score of 0,1 , or 2 points, depending on whether neither, one, or both made correct identifications based on

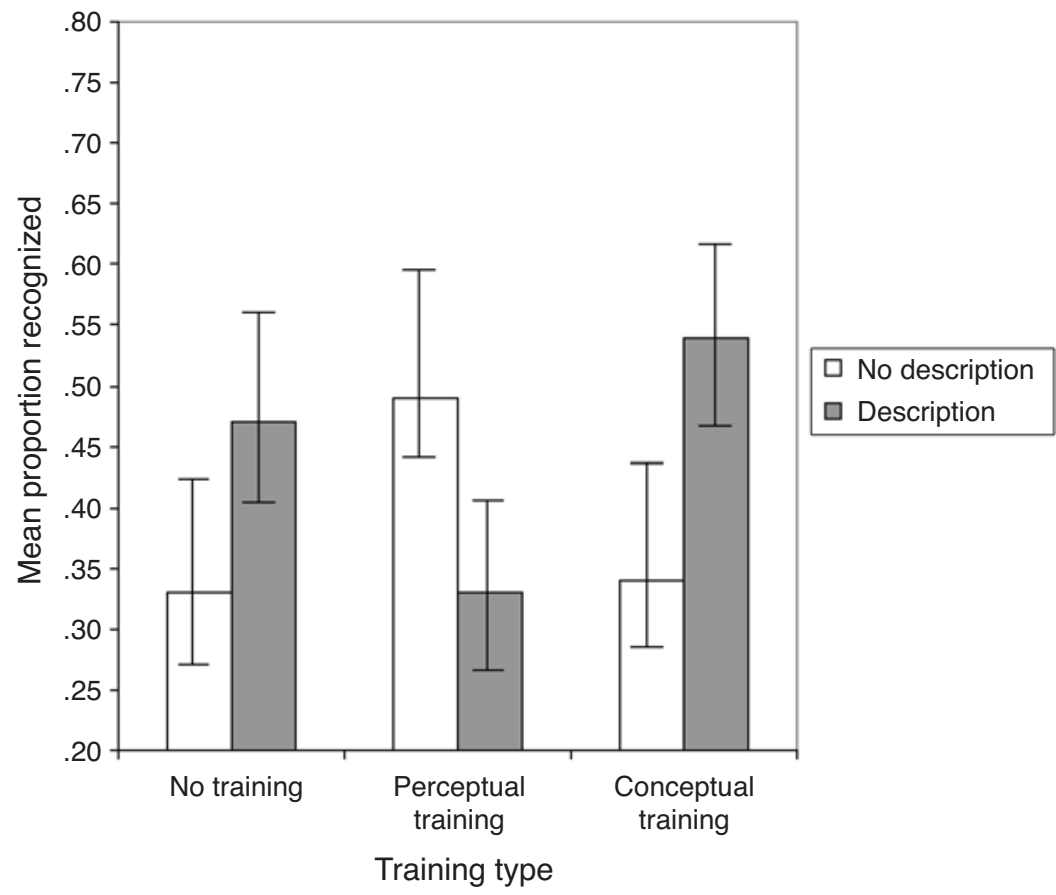

Figure 2. Trial 1 mean recognition accuracy as a function of training and verbalization. 


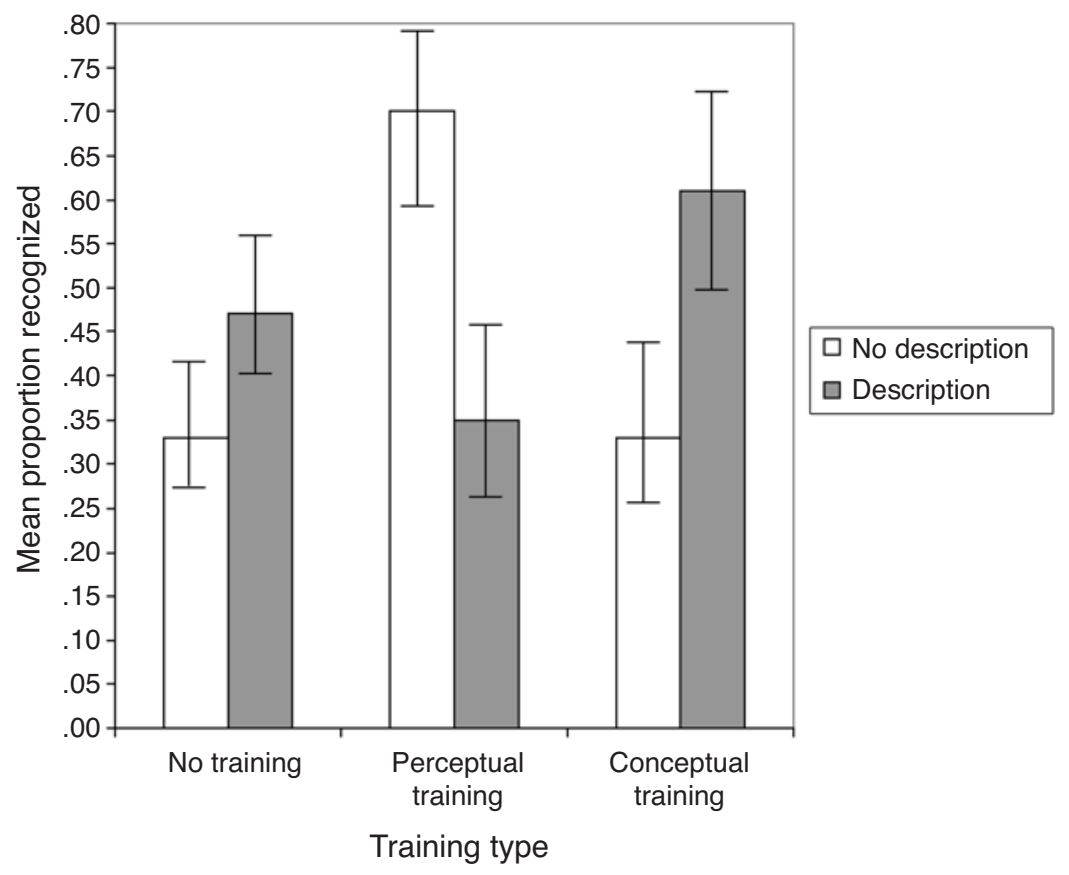

Figure 3. Trial 1 mean recognition accuracy (above-median participants only).

the descriptions. These scores were correlated with the original describer's accuracy ( 0 or 1$)$.

As seen in Table 1, the no training yoked judges had chance-level accuracy, whereas the conceptual and per- ceptual yoked judges had virtually identical (and abovechance) accuracy which was significantly higher recognition than that of the no training yoked judges $[F(2,237)=$ $3.89, p<.05]$. There was no correlation between original

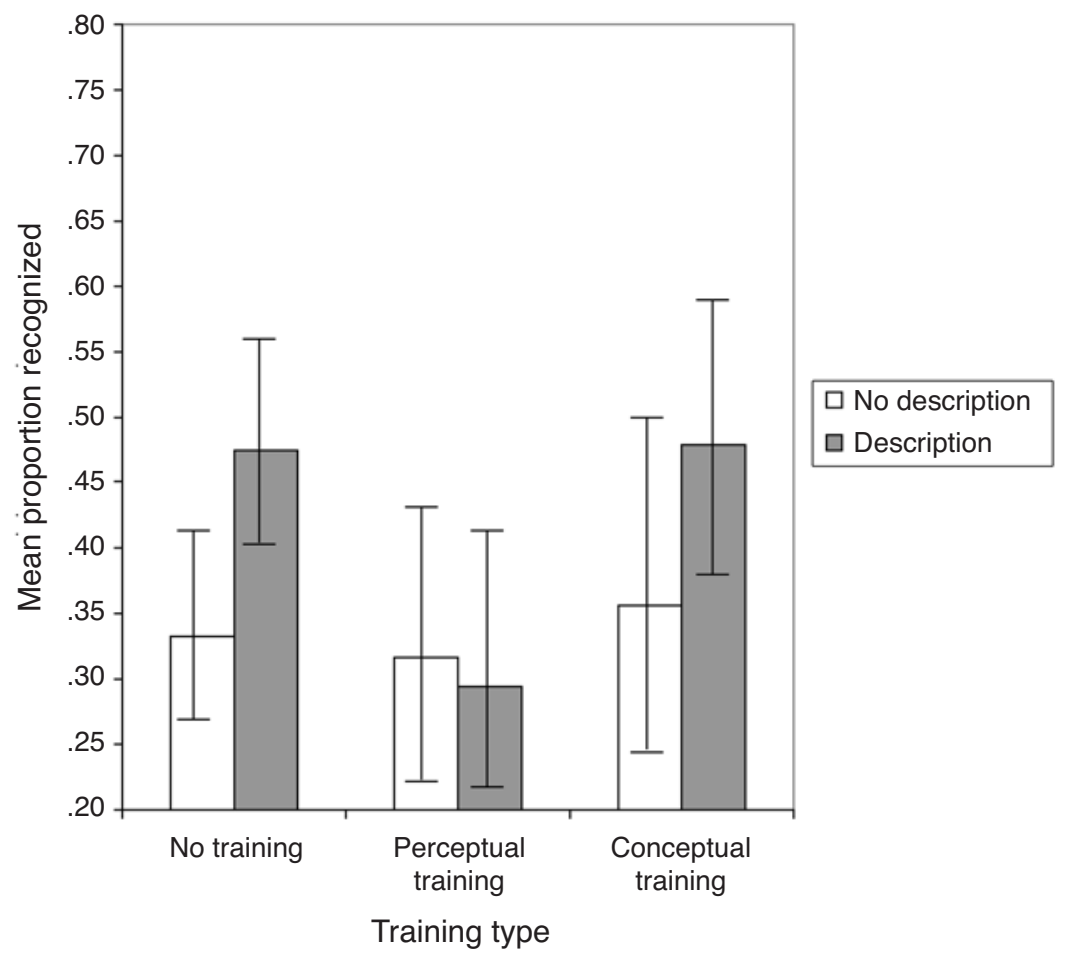

Figure 4. Trial 1 mean recognition accuracy (below-median participants only). 
Table 1

Mean Recognition Accuracies of, and Correlations Between, Original Describers and Yoked Judges

\begin{tabular}{lcc}
\hline \multicolumn{1}{c}{ Condition } & Mean Recognition & Pearson's $r$ \\
\hline No training & & \\
Original $(n=38)$ & .47 & -.08 \\
Yoked judges $(n=76)$ & .13 & \\
Perceptual training & & $.22 *$ \\
$\quad$ Original $(n=40)$ & .33 & \\
$\quad$ Yoked judges $(n=80)$ & .30 & \\
Conceptual training & & \\
Original $(n=40)$ & .54 & \\
Yoked judges $(n=80)$ & .29 & \\
$*_{p}=.05$ & &
\end{tabular}

$* p=.05$

and yoked judges in the no training and conceptual conditions, but there was a low, significant correlation in the perceptual condition.

\section{Differences Between Experiments 1 and 2}

Because Experiments 1 and 2 used very similar procedures with very similar participant populations, and because of the different pattern of results in the conceptual training condition relative to the other conditions, we deemed it worthwhile to compare performance across the two experiments. Although such analyses must be viewed with caution, they nevertheless proved potentially informative.

Perceptual training. As anticipated, the more intensive categorization training in Experiment 2 appeared to result in greater classification accuracy on the last third of the learning trials $(M=.78$, median $=.75)$ versus .73 and .72 in Experiment 1 . The increase was significant $[t(220)=$ $1.90, p=.048]$.

Conceptual training. Participants in Experiment 2 scored only 0.75 points higher on the mushroom quiz than they did in Experiment $1(M=11.3$ points, $S D=3.43)$; the difference was not significant $[t(255)=1.34, p=$ .18]. The above-median participants' quiz scores showed approximately the same increase, with means of 14.05 (Experiment 1) and 14.75 (Experiment 2).

We had predicted that our modified note-taking instructions would encourage participants to write more extensive notes than they did in Experiment 1 and thereby encourage them to generate a knowledge representation more commensurate with the verbalization task. To make the comparison, one of us (J.M.M.) used the conceptual training outline as the standard against which to do blind ratings of participants' notes, using a scale from 0 to $3(0=$ no notes, $1=$ minimal or very incomplete notes, $2=$ moderately detailed and complete notes, 3 = exceptionally detailed and complete notes). The mean note quality scores were 1.24 and 1.96 points for Experiments 1 and 2, respectively, and the difference was significant $[t(255)=$ $5.42, p=.00]$. This difference in note quality also reflects the fact that $28 \%$ of Experiment 1 participants wrote no notes (compared with only $13 \%$ in Experiment 2 ). The difference was significant $\left[\chi^{2}(1, N=30)=4.20, p=\right.$ $.04]$. To be conservative, we reanalyzed this data after excluding participants who wrote no notes. But Experiment 2 note quality scores ( $M=2.24$ points) still significantly exceeded those from Experiment 1 ( $M=1.72$ points) $[t(199)=4.61, p=.00]$. Thirty-nine percent of Experiment 2 participants wrote highest quality notes compared with only $16 \%$ of Experiment 1 participants. This difference was also significant $\left[\chi^{2}(1, N=49)=7.36, p=\right.$ .007]. Thus, it seems clear that the modified instructions encouraged more, and higher quality, note taking.

We had hypothesized that participants who took lower quality notes would be more susceptible to verbal overshadowing because less effort in verbalizing should produce less disparity between verbal and perceptual representations at recognition. In a post hoc test of this hypothesis, we split the note-taking scores from both experiments into low and high groups on the basis of the note quality ratings $(0 / 1,2 / 3)$. In Experiment 2, high-quality note takers in the description condition achieved higher recognition than did those in the no description group (55\% vs. $39 \%$, a verbal enhancement effect). In contrast, among lowquality note takers, the no description controls had higher recognition than did the describers ( $55 \%$ vs. $47 \%$, a verbal overshadowing effect). Although this pattern is consistent with our explanation for the results of Experiment 2, an apparent note quality $\times$ verbalization interaction was not significant $\left[\chi^{2}(1, N=158)=1.64, p=.20\right]$.

Finally, it is worth noting that in Experiment 1, the conceptual training no description participants had much higher recognition than did their counterparts in Experiment $2(M \mathrm{~s}=.47$ and .33 , respectively; see Figures 1 and 2$)$. This difference is consistent with our conjecture that the Experiment 1 conceptual training participants paid less attention to the conceptual components and, by default, more attention to the "contaminating" perceptual aspects of the training. Thus, Experiment 1 participants may have done relatively well on the recognition when they did not describe because their processing at recognition was similar to their processing during learning.

\section{Discussion}

Experiment 2 replicated the Experiment 1 result that individuals who received perceptual training in an unfamiliar perceptual domain were more susceptible to verbal overshadowing effects than were individuals who received no such training. In addition, it produced the originally anticipated (though not observed) interaction between verbalization and training. Specifically, in contrast to participants who received perceptual training, those who received conceptual training with note taking did not show verbal overshadowing of mushroom recognition. Indeed, although only marginally significant, conceptually trained participants who verbalized appeared to benefit from their training.

Although we must be cautious in comparing across experiments, the different impact of the conceptual training in Experiments 1 and 2 was likely due to the fact that Experiment 2 more explicitly encouraged note taking. These participants were more likely to take notes than were their Experiment 1 counterparts, and their notes were of higher quality. Given that this resulted in significantly different 
note-taking behavior, it seems quite plausible that this innovation produced the different results within the conceptual condition in Experiment 2.

The communication accuracy data show that, compared with no training, perceptual and conceptual training enabled participants to write at least moderately communicative descriptions that were equally useful to their yoked judges. However, the conceptual describers, while notably more accurate in their own recognition, did not generate descriptions that were any more informative than those generated by the perceptual describers. One possible explanation for the lack of a difference between the identification accuracy of conceptually and perceptually trained participants' yoked judges is that the yoked judges would not have understood at least some featural information in the descriptions (i.e., mushroom vocabulary). Alternatively, it may be that the advantage of conceptual training is not that participants generate more accurate descriptions per se, but rather that they learn to process the stimuli in a verbal/conceptual manner that is more commensurate with the process of verbal description.

\section{GENERAL DISCUSSION}

The results of these two studies demonstrate that the effects of verbal overshadowing depend on both the extent and the type of experience with a stimulus domain. In both experiments, perceptually trained recognition performance was impaired by verbalization. In contrast, participants who received no training were not significantly affected by verbalization, and those who received conceptual training apparently benefited from verbalization in Experiment 2 , in which the procedure had been refined to maximize conceptual learning.

The observation that perceptual training increases individuals' vulnerability to verbalization is consistent with other recent findings demonstrating that individuals with superior perceptual expertise in a domain are more vulnerable to verbalization than are individuals with less expertise (e.g., own-race face recognition, Diamond \& Carey, 1986; Fallshore \& Schooler, 1995; or wine tasting, Melcher $\&$ Schooler, 1996). However, in contrast to these studies, the present experiments demonstrated this pattern with a newly acquired rather than preexisting expertise. Thus, we can be more confident that it was perceptual experience and not some correlate of expertise that mediated the verbal overshadowing effect. Additional evidence for the importance of perceptual experience in mediating the verbal overshadowing effect comes from a comparison of the effects of verbalization on participants who performed above, versus below, the median on the original perceptual training procedure. This analysis revealed that participants who performed above the median on their respective training procedures benefited most from their training. Most important, this benefit of successful perceptual training was lost when perceptually trained participants were forced to verbalize their knowledge prior to recognition.

In contrast to performance in the perceptual and no training conditions, that in the conceptual training condi- tion was inconsistent between the two studies. Whereas conceptually trained participants in Experiment 1 showed a numeric verbal overshadowing, their counterparts in Experiment 2 revealed a nearly significant verbal enhancement. Although resolution of this discrepancy requires comparing the procedures across experiments, this pattern of findings can be readily accommodated on the assumption that the training in Experiment 1 failed to adequately emphasize conceptual over perceptual training. Whereas there was no possible conceptual contamination in the perceptual training, it was impossible to avoid perceptual exposure in the conceptual training condition. Given that Experiment 2 participants apparently put more effort into taking notes, they may have acquired a higher ratio of conceptual to perceptual knowledge than did those in Experiment 1 . That is, conceptual training participants in both experiments may have acquired approximately the same amount of perceptual learning (i.e., contamination), but gained relatively more conceptual knowledge in Experiment 2 .

\section{Conceptualizing the Interaction \\ Between Verbal Overshadowing and Expertise}

In prior discussions of the verbal overshadowing effect, two basic accounts have been promoted. According to a recoding interference view (Meissner, 2002; Meissner, Brigham, \& Kelley, 2001; Schooler \& Engstler-Schooler, 1990) verbalization generates a new verbal representation that distorts or misrepresents the original memory, thereby serving as a source of interference. Alternatively, according to a processing shift account (Dodson, Johnson, \& Schooler, 1997; Fallshore \& Schooler, 1995; Schooler, 2002; Schooler, Fiore, \& Brandimonte, 1997; Schooler, Ryan, \& Reder, 1996; Westerman, 1997), verbalization causes a general shift in the manner in which individuals process the critical stimuli. Like standard transfer-appropriate processing accounts of memory (e.g., Morris, Bransford, \& Franks, 1977), this "transfer-inappropriate" processing shift account assumes that stimuli that are encoded nonverbally will be best recognized if similar nonverbal processes are drawn upon during recognition. If, however, individuals are encouraged to engage in verbal retrieval between encoding and recognition, then this verbal processing may be inappropriately carried over to recognition, hampering the application of perceptual recognition processes, causing the individuals to emphasize nondistinguishing verbal information while deemphasizing critically applicable nonverbal information. In principle, the present findings could be interpreted within either a recoding interference account or a processing shift account.

According to a recoding interference account, the advantage of individuals trained with conceptual information over those with perceptual information is that the latter developed a vocabulary that enabled them to describe their experiences in a manner that did not produce selfgenerated misinformation. The fact that in Experiment 2 a correlation between description quality (as measured by yoked judges) and recognition performance was exclusively observed in the condition in which verbal over- 
shadowing was evidenced (i.e., the perceptual training condition) also is consistent with the possibility that poor descriptions led to poor recognition performance (for a similar argument, see Meissner et al., 2001). However, this correlation was extremely modest, accounting for less than $5 \%$ of the variance, and therefore we must be cautious in assuming that variations in description accuracy were the source of the verbal overshadowing effect.

According to a processing shift account, perceptually trained describers were impaired because they first encoded the target using the implicit, nonverbal, nonanalytic process encouraged by the training. They switched to explicit, verbal, analytical processing to write their descriptions, but then failed to revert to the original mode for the recognition decision, and thus lacked the critical nonverbal sources of information that they had originally used to encode the information. In contrast, the conceptually trained participants, having already learned to process the stimuli in a more verbal manner, experienced no discrepancy between the operations used at encoding and those applied after verbalization, and therefore experienced no detriment (at least in Experiment 2). Indeed, this processing shift account helps to explain the unexpected verbal overshadowing that we observed in the conceptual group of Experiment 1. Accordingly, various measures (e.g., differential note-taking performance) suggest that participants in the conceptual training condition did not engage as fully in conceptual learning in Experiment 1, relative to Experiment 2. Thus, participants in the conceptual conditions of Experiment 1 may have been more likely to process the target mushroom perceptually (given the perceptual aspects of the conceptual training) and therefore were vulnerable to the inappropriate processing shift that similarly plagued their perceptually trained counterparts.

A processing shift account can also help explain why the descriptions generated by perceptually trained participants were as useful to the yoked judges as were those generated by their conceptually trained counterparts, despite the former group's 21-point lower recognition accuracy. From a recoding interference perspective, we would expect that perceptually trained participants' lower recognition accuracy would be associated with poorer description quality. Instead, the very similar identification rates of the yoked judges in the two conditions suggest that the advantage of the training was not necessarily to generate better descriptions but rather to encourage participants to encode the stimuli in a manner consistent with the processes invoked during verbalization and recognition.

Finally, the attenuation of the verbal overshadowing effect over trials, found here and elsewhere, can be generally understood with the transfer-inappropriate retrieval framework: Having gone through the entire procedure, participants may become more consistent in the processes that they apply during encoding, verbalization, and test. The increased alignment of processes may thereby reduce the likelihood that processes applied at retrieval will be inconsistent with those applied earlier.

In short, while in principle either the recoding interference or processing shift accounts might explain the pres- ent findings, the processing shift account explains a greater number of distinct results. It also should be noted that the processing shift account accommodates several findings not well handled by the recoding interference view. These include the findings that (1) describing one stimulus can interfere with recognition of a nondescribed stimulus (Brown \& Lloyd-Jones, 2002; Dodson et al., 1997); (2) there is frequently a very small or nonexistent relationship between the quality of verbal descriptions and that of recognition accuracy (e.g., Brown \& LloydJones, 2002, 2003; Fallshore \& Schooler, 1995; (3) verbal overshadowing-like effects can be induced simply by engaging participants in featural versus configural processes between encoding and test (Macrae \& Lewis, 2002); and (4) verbal overshadowing effects can be reversed by manipulations designed to reinstate the perceptual mode of processing, including re-presenting the face (Schooler et al., 1996), reinstating visual cues (Brandimonte, Schooler, \& Gabbino, 1997), or engaging participants in a perceptual task such as maze completion or listening to music before the recognition test (Finger, 2002). For a more extensive, recent review of evidence that verbalization induces a transfer-inappropriate processing shift, see Schooler (2002)..$^{5}$

\section{Nature of the Processes That Are Disrupted by Verbalization}

The claim that the present results, and those of prior verbal overshadowing studies, reflect a conflict between more verbal versus more perceptual processes, begs the question of whether the nature of these processes can be more precisely specified. We have previously speculated that verbalization may encourage more featural analytic processing, at the expense of more configural nonanalytic processing. Evidence for this claim comes from the fact that verbalization disrupts various tasks known to be associated with holistic processing (e.g., own-race and upright-face recognition) while having less impact on tasks that rely on featural processing (e.g., other-race and inverted-face recognition) (Fallshore \& Schooler, 1995). A featural processing shift is also implicated in recent findings (Macrae \& Lewis, 2002) demonstrating that verbal overshadowing-like effects can be produced simply by encouraging featural versus configural processes between encoding and test, by showing participants text composed of smaller letters and having them either attend to the small letters (featural processing) or the large letters (configural processing). In the present case, the two training procedures also varied in their emphasis on featural versus configural processing, with the conceptual training procedure explicitly teaching participants to verbally identify individual features, and the perceptual training exclusively focusing on entire mushrooms. Thus, it seems likely that a key difference between the perceptually and conceptually trained participants was the degree to which they spontaneously emphasized featural versus configural processing.

Although the featural/configural processing distinction is one important way to conceptualize the conflicting 
processes observed in the present study, it is not the only one. For example, the conceptual training condition (with its explicit instructions) presumably engaged more explicit processes, whereas the perceptual training condition, which provided no explicit instructions, may have engaged more implicit learning processes. Strategic versus automatic processes (e.g., Johnson \& Hasher, 1987) and reflective versus nonreflective processes (e.g., Hayes \& Broadbent, 1988; Schooler, 2002) might also have been differentially engaged in the two training procedures. And of course, verbal versus nonverbal processes were likely to have been differentially emphasized in the two conditions, particularly in Experiment 2, in which the most substantial difference between the training conditions was observed. Although future research might profitably attempt to further tease out which of these related sets of processes are critical to producing the differential effects of training, many of these processes appear to overlap in fundamental ways. Verbal analysis seems likely to inevitably lead to more featural, strategic, and explicit analysis than does training that merely emphasizes perceptual apprehension. Thus, it seems likely that the distinct processes emphasized by the respective training procedures may have involved two constellations of overlapping mechanisms: one emphasizing verbal, featural, conceptual, strategic, and explicit processes and the other emphasizing perceptual, configural, nonstrategic, and implicit processes.

\section{REFERENCES}

Ahissar, M. (1999). Perceptual learning. Current Directions in Psychological Science, 8, 124-128.

Ahissar, M., \& Hochstein, S. (1997). Task difficulty and the specificity of perceptual learning. Nature, 387, 401-406.

Brandimonte, M. A., Hitch, G. J., \& Bishop, D. V. M. (1992). Verbal recoding of visual stimuli impairs mental image transformations. Memory \& Cognition, 20, 449-455.

Brandimonte, M. A., Schooler, J. W., \& Gabbino, P. (1997). Attenuating verbal overshadowing through visual retrieval cues. Journal of Experimental Psychology: Learning, Memory, \& Cognition, 23, 915-931.

Brown, C., \& Lloyd-Jones, T. J. (2002). Verbal overshadowing in a multiple face presentation paradigm: Effects of description instruction. Applied Cognitive Psychology, 16, 873-885.

Brown, C., \& Lloyd-Jones, T. J. (2003). Verbal overshadowing of multiple face and car recognition: Effects of within- versus acrosscategory verbal descriptions. Applied Cognitive Psychology, 17, 183201.

DeShon, R. P., Chan, D., \& Weissbein, D. A. (1995). Verbal overshadowing effects on Raven's Advanced Progressive Matrices: Evidence for multidimensional performance determinants. Intelligence, 21, 135-155.

Diamond, R., \& CARey, S. (1986). Why faces are and are not special: An effect of expertise. Journal of Experimental Psychology: General, 115, 107-117.

Dodson, C. S., Johnson, M. K., \& Schooler, J. W. (1997). The verbal overshadowing effect: Why descriptions impair face recognition. Memory \& Cognition, 25, 129-139.

FALlshore, M., \& SCHOOLER, J. W. (1995). Verbal vulnerability of perceptual expertise. Journal of Experimental Psychology: Learning, Memory, \& Cognition, 21, 1608-1623.

FINGER, K. (2002). Mazes and music: Using perceptual processing to release verbal overshadowing. Applied Cognitive Psychology, 16, 887-896.

Fiore, S. M., \& Schooler, J. W. (2002). How did you get here from there? Verbal overshadowing of spatial mental models. Applied Cognitive Psychology, 16, 897-909.
Goldstone, R. L. (1998). Perceptual learning. Annual Review of Psychology, 49, 585-612.

HAIDER, P. A., \& FRENSCH, H. (1996). The role of information reduction in skill acquisition. Cognitive Psychology, 30, 304-337.

Hall, G. (1991). Perceptual and associative learning. Oxford: Oxford University Press, Clarendon Press.

Hayes, N. A., \& Broadbent, D. (1988). Two modes of learning for interactive tasks. Cognition, 28, 249-276.

Holyoak, K. J., \& Spellman, B. A. (1993). Thinking. Annual Review of Psychology, 44, 265-315.

Houser, T., Fiore, S. M., \& Schooler, J. W. (2003). Verbal overshadowing of music memory: What happens when you describe that tune? Unpublished manuscript.

Johnson, M. K., \& Hasher, L. (1987). Human learning and memory. Annual Review of Psychology, 38, 631-668.

Levy, B. J., \& Anderson, M. C. (2002). Inhibitory processes and the control of memory retrieval. Trends in Cognitive Sciences, 6, 299-305.

Mackintosh, N. J. (1995). Categorization by people and pigeons: The twenty-second Bartlett Memorial Lecture. Quarterly Journal of Experimental Psychology, 48B, 193-214.

Macrae, N. C., \& Lewis, H. L. (2002). Do I know you? Processing orientation and face recognition. Psychological Science, 13, 194-196.

McLaren, I. P. L., Leevers, H. J., \& Mackintosh, N. J. (1994). Recognition, categorization, and perceptual learning (or, how learning to classify things together helps one to tell them apart). In C. Umiltà \& M. Moscovitch (Eds.), Attention and performance XV: Conscious and nonconscious information processing (pp. 889-909). Cambridge, MA: MIT Press, Bradford Books.

Meissner, C. A. (2002). Applied aspects of the instructional bias effect in verbal overshadowing. Applied Cognitive Psychology, 16, 911-928.

Meissner, C. A., \& Brigham, J. C. (2001). A meta-analysis of the verbal overshadowing effect in face identification. Applied Cognitive Psychology, 15, 603-616.

Meissner, C. A., Brigham, J. C., \& Kelley, C. M. (2001). The influence of retrieval processes in verbal overshadowing. Memory \& Cognition, 29, 176-186.

Melcher, J. M. (2003). Mushroom reference texts and sample images. Available at web.stcloudstate.edu/jmmelcher/mushrefs.htm.

Melcher, J. M., \& Schooler, J. W. (1996). The misremembrance of wines past: Verbal and perceptual expertise differentially mediate verbal overshadowing of taste memory. Journal of Memory \& Language, 35, 231-245.

Morris, C. D., Bransford, J. D., \& Franks, J. J. (1977). Levels of processing versus transfer appropriate processing. Journal of Verbal Learning \& Verbal Behavior, 16, 519-533.

Perfect, T. J., Hunt, L. J., \& Harris, C. M. (2002). Verbal overshadowing in voice recognition. Applied Cognitive Psychology, 16, 973980.

Psychology Software Tools (1993). MEL Lab: Experiments in perception, cognition, social psychology, \& human factors [Computer software]. Pittsburgh, PA: Psychology Software Tools.

REBer, A. S. (1993). Implicit learning and tacit knowledge: An essay on the cognitive unconscious. New York: Oxford University Press, Clarendon Press.

Rhodes, G., Brake, S., \& AtKinson, A. P. (1993). What's lost in inverted faces? Cognition, 47, 25-57.

RYAN, R., \& SCHOOLER, J. W. (1998). Whom do words hurt? Individual differences in susceptibility to verbal overshadowing. Applied Cognitive Psychology, 12, S105-S125.

SCHOOLER, J. W. (2002). Verbalization produces a transfer inappropriate processing shift. Applied Cognitive Psychology, 16, 989-997.

SCHOOLER, J. W., \& EngSTLER-SchoOler, T. Y. (1990). Verbal overshadowing of visual memories: Some things are better left unsaid. Cognitive Psychology, 22, 36-71.

Schooler, J. W., Fiore, S. M., \& Brandimonte, M. A. (1997). At a loss from words: Verbal overshadowing of perceptual memories. In D. L. Medin (Ed.), The psychology of learning and motivation (Vol. 37, pp. 293-334). San Diego: Academic Press.

Schooler, J. W., OHLsson, S., \& Brooks, K. (1993). Thoughts beyond words: When language overshadows insight. Journal of Experimental Psychology: General, 122, 166-183. 
Schooler, J. W., Ryan, R. S., \& Reder, L. (1996). The costs and benefits of verbally rehearsing memory for faces. In D. Herrmann, M. Johnson, C. McEvoy, C. Hertzog, \& P. Hertel (Eds.), Basic and applied memory: New findings (pp. 51-65). Mahwah, NJ: Erlbaum.

SieCK, W. R., QuinN, C. N., \& SCHOOLER, J. W. (1999). Justification effects on the judgment of analogy. Memory \& Cognition, 27, 844-855.

Tetlock, P. E., \& Kim, J. I. (1987). Accountability and judgment processes in a personality prediction task. Journal of Personality \& Social Psychology, 52, 700-709.

Westerman, D. (1997). The verbal overshadowing effect: Evidence for a general shift in processing. American Journal of Psychology, 110, 417-428.

Wilson, T. D., \& Schooler, J. W. (1991). Thinking too much: Introspection can reduce the quality of preferences and decisions. Journal of Personality \& Social Psychology, 60, 181-192.

\section{NOTES}

1. In a mutilated checkerboard, the black and white squares are randomly rearranged to form a prototype. Participants learn about the prototype by viewing numerous patterns that vary from the prototype in small random ways.

2. There are several possible explanations for this trial effect. Fallshore and Schooler (1995) postulated that individuals may learn to improve the quality of their face descriptions or, alternatively, may learn to ignore their descriptions. Using a communication-accuracy paradigm (Ex- periment 2), they found no evidence that participants were ignoring their descriptions, but did find tentative evidence that descriptions had improved over trials. Whatever causes this trial effect, it very probably involves a strategy shift reflecting increasing sensitivity to task demands (cf. Haider \& Frensch's, 1996, demonstration of practice effects in which individuals shift strategies on the basis of a task's structural demands). For example, participants may learn to encode the stimulus in a manner more commensurate with the task of verbal description or to discount their verbalization activity.

3. Coincidentally, $72 \%$ is almost exactly the level indicating significantly above-chance performance in a binomial test, given 18 trials.

4. As both here and in Experiment 1, Melcher and Schooler (1996) observed a similar (and also nonsignificant) trend toward a benefit of verbalization among wine novices (equivalent to the present no training condition).

5. Schooler et al. (1997) proposed a possible link between transferinappropriate processing and retrieval-induced inhibition in accounting for the verbal overshadowing effect. In retrieval-induced inhibition, retrieval of one item can inhibit memory for related, but previously unrecalled, items (Levy \& Anderson, 2002). Thus, Schooler et al. (1997) suggested that retrieval involving one process (e.g., explicit, verbal) might inhibit later use of implicit, nonverbal processes on a subsequent memory test. Although such retrieval-specific inhibition processes may be involved in verbal overshadowing, we do not emphasize them here due to recent evidence that seemingly comparable processing shifts can be induced without memory retrieval (e.g., Macrae \& Lewis, 2002). 
APPENDIX A

Sample Perceptual Training (Classification) Photographs

(Reproduced in grayscale; color photos and references available at: web.stcloudstate.edu/jmmelcher/mushrefs.htm)

Genus Agaricacea
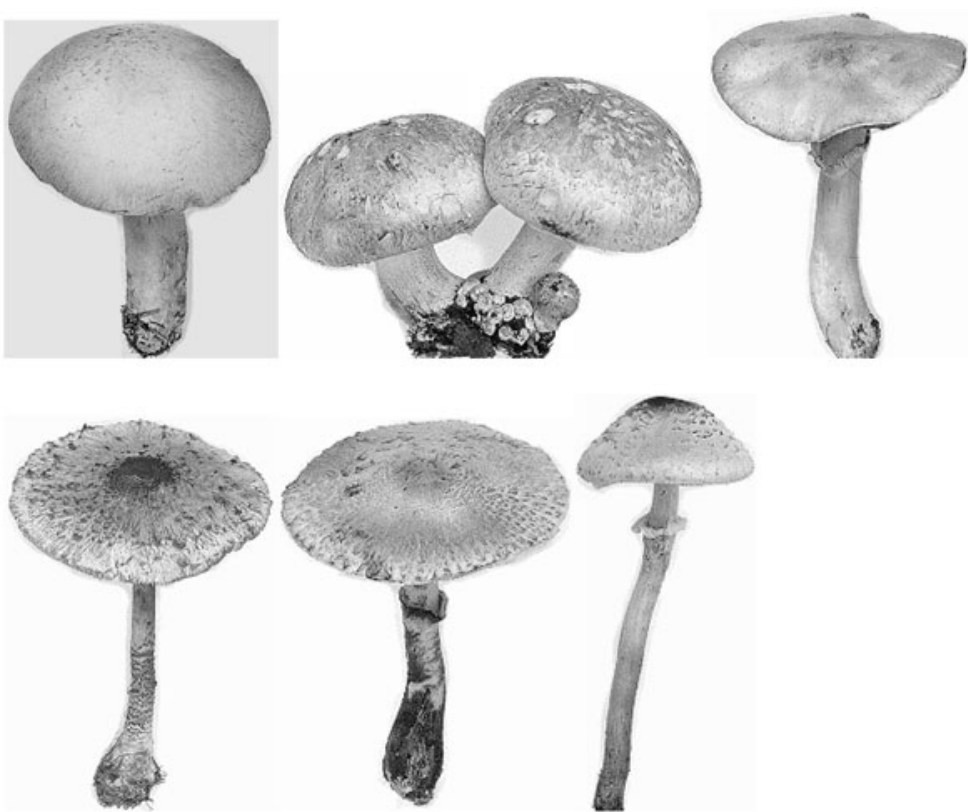

Genus Amanitacea
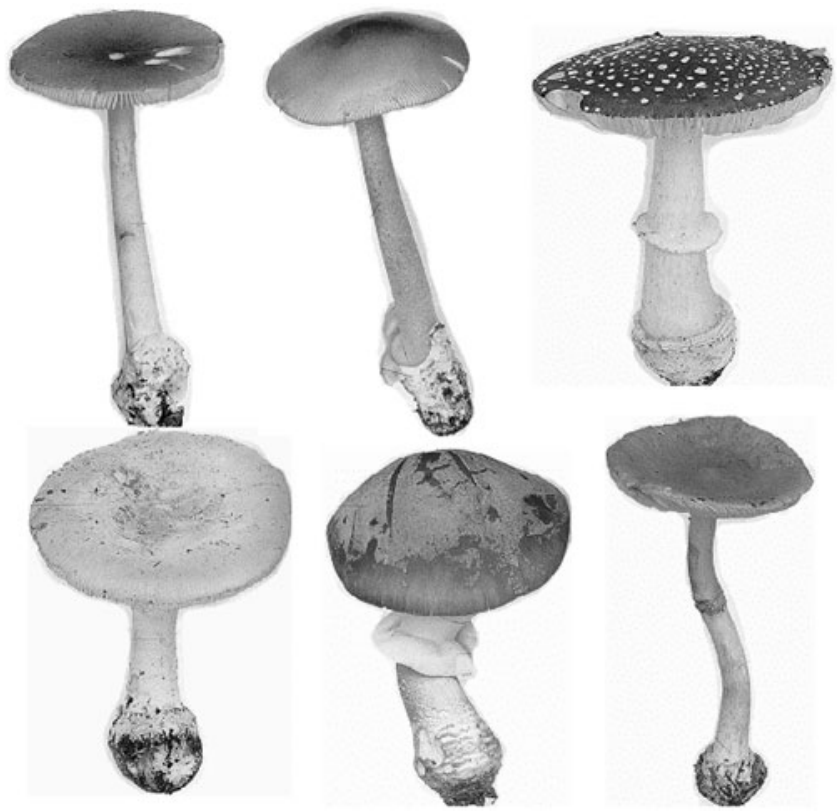
APPENDIX B

Sample Mushroom Target Photos and Recognition Arrays

(Color photos and references available at web.stcloudstate.edu/jmmelcher/mushrefs.htm)

Lepiota cristata target and recognition array
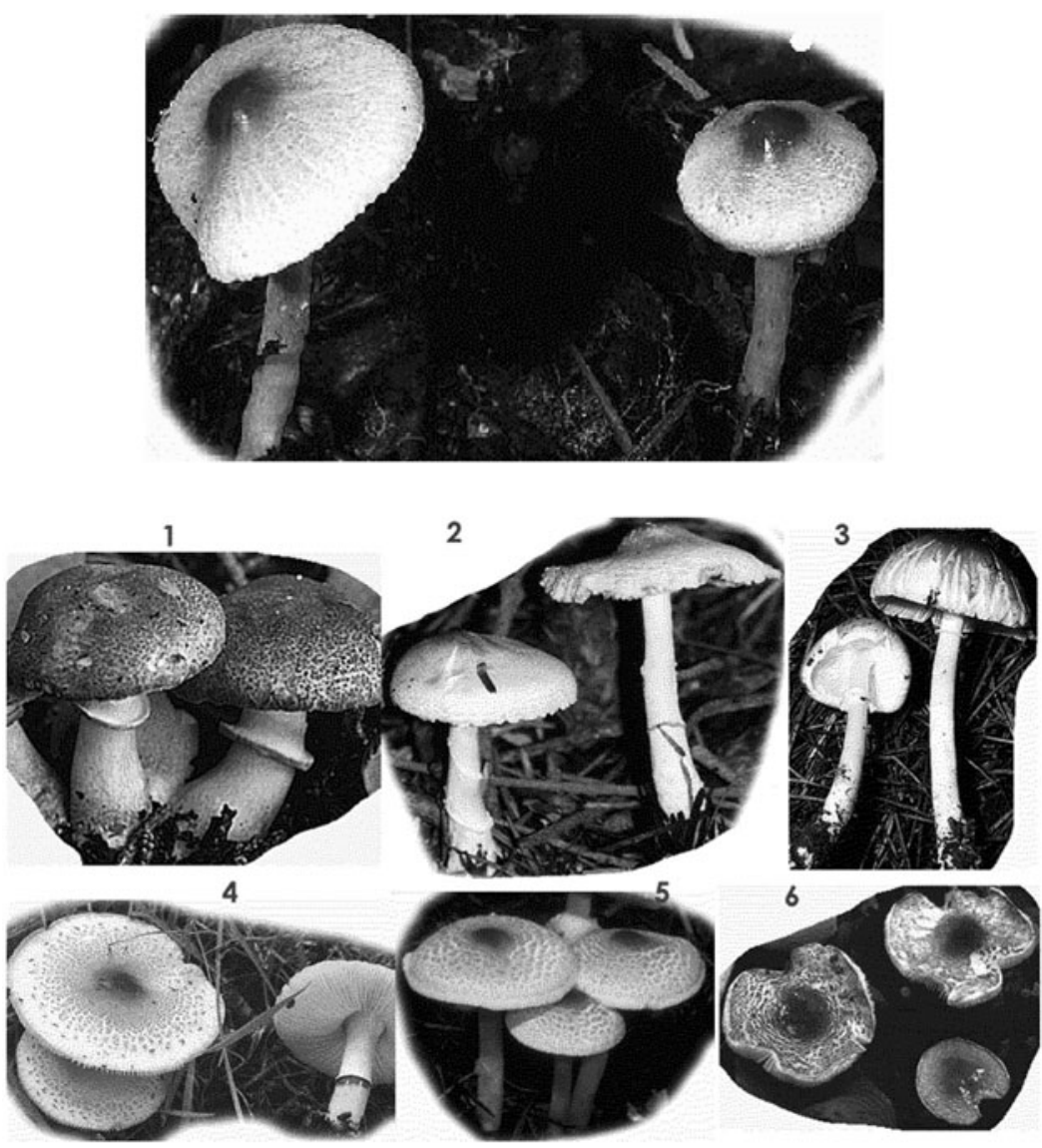

(Manuscript received October 15, 2002;

revision accepted for publication December 2, 2003.) 